\title{
Analysis of the Status and Influencing Factors of Failed Ultrasound-guided Invasive Arterial Catheterization in ICU Patients
}

\author{
Mei Feng ${ }^{1,3}$, Ni Zhang ${ }^{3}$, Miaohang Shan ${ }^{1}$, Yanbin Pan ${ }^{1}$, Wanxian Lu ${ }^{1, ~ *, ~ Q i n g r a n ~ L i n ~}{ }^{2, *}$ \\ ${ }^{1}$ Department of Intensive Medicine, the First Affiliated Hospital of Jinan University, Guangzhou, China \\ ${ }^{2}$ Department of Nursing, the First Affiliated Hospital of Jinan University, Guangzhou, China \\ ${ }^{3}$ College of Nursing, Jinan University, Guangzhou, China
}

Email address:

fm1234567892020@126.com (Mei Feng),765277753@qq.com (Wanxian Lu), qingranlin@126.com (Qingran Lin)

${ }^{*}$ Corresponding author

\section{To cite this article:}

Mei Feng, Ni Zhang, Miaohang Shan, Yanbin Pan, Wanxian Lu, Qingran Lin. Analysis of the Status and Influencing Factors of Failed Ultrasound-guided Invasive Arterial Catheterization in ICU Patients. Clinical Medicine Research. Vol. 9, No. 6, 2020, pp. 140-144. doi: $10.11648 /$ j.cmr.20200906.14

Received: December 14, 2020; Accepted: December 22, 2020; Published: December 31, 2020

\begin{abstract}
Background: Catheter failure means that the arterial catheter loses its original real-time and accurate function of monitoring the blood pressure due to various causes, such as blockage, buckling, displacement of the catheter, edema, bleeding, and oozing of the punctured limb requiring catheter removal. However, there have been few studies about the catheter failure and its related influencing factors. The present study aims to explore the status and influencing factors of failed ultrasound-guided invasive arterial catheterization in ICU patients. Methods: 264 patients who underwent ultrasound-guided invasive arterial catheterization from January 2019 to December 2019 were analyzed retrospectively to observe the status and failure of the arterial catheterization. Statistical analysis was performed using SPSS22.0 software. Results: Among the 264 cases with invasive arterial catheterization, 59 cases had catheter failure, the incidence was $22.34 \%$. Single factor analysis showed that the use of analgesics, the protective catheter sleeve, and the affected limb restraints could prevent catheter failure significantly $(\mathrm{P}<0.05)$. The result of multivariate logistic regression analysis showed that the affected limb restraints, and the use of analgesics were protective factors against the failure of invasive arterial catheterization $(\mathrm{P}<0.05)$, with $\mathrm{OR}$ values of 0.454 and 0.450 , respectively. Conclusion: Under ultrasound guidance, the incidence of catheter failure after invasive arterial catheterization is low. For the ICU patients undergoing invasive arterial catheterization, analgesics should be used, affected limbs should be constrained, and health education, and communication should be conducted to further reduce the incidence of catheter failure.
\end{abstract}

Keywords: Intensive Care Unit, Ultrasound Guidance, Invasive Arterial Catheterization, Catheter Failure, Influencing Factors

\section{Introduction}

Invasive arterial catheterization has been widely used in dynamic monitoring of patients' vital signs for ICU patients $[1,2]$. Success rate is improved in placement of invasive arterial catheters under ultrasound guidance [3] with reduction of complications caused by invasive arterial catheterization [4-7]. However, there have been few studies about the catheter failure and its related influencing factors. Catheter failure means that the arterial catheter loses its original real-time and accurate function of monitoring the blood pressure due to various causes, such as blockage, buckling, displacement of the catheter, edema, bleeding, and oozing of the punctured limb requiring catheter removal [8]. In this study, a retrospective analysis of 264 patients who underwent invasive arterial catheterization under the guidance of ultrasound from January 2019 to December 2019 in the Department of Intensive Medicine of our hospital for in-depth study of the related influencing factors leading to catheter failure to effectively reduce the incidence, and provide a practical basis for the standardization of clinical catheter placement and maintenance. 


\section{Data and Methods}

\subsection{General Data}

264 patients who underwent ultrasound-guided invasive arterial catheterization from January 2019 to December 2019 in our hospital were selected. There were 193 males and 71 females. The average age was $62.23 \pm 18.101$ years (range, 8-94 years).

\subsection{Methods}

Preoperative education was carried out for conscious patients. Ultrasound-guided invasive arterial puncture and catheterization was performed for all patients. After successful puncture, the catheter was fixed properly and connected to the monitor for invasive blood pressure monitoring. Complications such as edema, vasospasm, skin bruising, bleeding, and oozing of the affected limb, buckling and blockage of the catheter were observed dynamically.

\subsection{Methods of data Collection}

A self-made retrospective analysis questionnaire of our hospital was used to collect the data of all patients on the general condition, the presence of catheter failure and the cause of catheter failure by consulting clinical records and other clinical data. The main contents of the questionnaire include: (1) general condition of the patient: gender, age, disease diagnosis, APACHE II score, oxygen use, history of hypertension, history of diabetes, history of smoking, history of drinking, history of coronary heart disease; (2) catheterization-related materials: catheter fixing materials, type of the puncture needle; (3) site of catheter placement: right radial artery, left radial artery, right dorsalis pedis artery; (4) examination indicators: fibrin value, activated partial thromboplastin time value (APTT); (5) post-catheterization care: use of sedatives, analgesics, protective catheter sleeves, affected limb restraints; (6) observation indicators of catheter failure: edema, vasospasm, skin bruising, bleeding, and oozing around the puncture site, buckling and blockage of the catheter, etc.

\subsection{Statistical Analysis}

In single factor analysis, measurement data was expressed as Mean \pm SD. The $t$ test was used for the statistical analysis. The count data are expressed as frequency and percentage, and statistically analyzed by chi-square test. For multivariate analysis, binary logistic regression model was used. The variable selection was performed using the forward selection method, and the inclusion level wastwo-sided. $\mathrm{P}<0.05$ was considered statistically significant. All data were analyzed by SPSS 22.0 software.

Table 1. Single factor analysis of the occurrence of the catheter failure (cases, \%).

\begin{tabular}{|c|c|c|c|c|c|c|}
\hline Items & & Total number & Failure & Non-failure & Statistics & P value \\
\hline Age (years) & & & $63.58 \pm 20.17$ & $62.36 \pm 17.51$ & 0.4562 & 0.649 \\
\hline \multirow{2}{*}{ Gender } & Male & $193(73.1)$ & $41(21.2)$ & $152(78.8)$ & 0.5051 & 0.477 \\
\hline & Female & $71(26.9)$ & $18(25.4)$ & $53(74.6)$ & & \\
\hline GCS score & & & $6.66 \pm 4.24$ & $7.93 \pm 4.48$ & 1.7242 & 0.086 \\
\hline SAS score & & & $2.08 \pm 0.289$ & $2.30 \pm 0.614$ & 1.8002 & 0.080 \\
\hline APACHE II score & & & $19.32 \pm 6.94$ & $18.86 \pm 7.25$ & 0.4322 & 0.666 \\
\hline Indwelling time (hours) & & & $85.75 \pm 97.70$ & $96.73 \pm 66.42$ & 0.8112 & 0.420 \\
\hline Fibrin $(\mathrm{g} / \mathrm{L})$ & & & $4.23 \pm 2.14$ & $4.55 \pm 1.88$ & 1.1102 & 0.268 \\
\hline \multirow[t]{2}{*}{ APTT (seconds) } & & & $49.71 \pm 23.22$ & $49.00 \pm 20.71$ & 0.2252 & 0.822 \\
\hline & Right radial artery & $226(85.6)$ & 49 (21.7) & $177(78.3)$ & 2.3122 & 0.315 \\
\hline \multirow[t]{2}{*}{ Puncture site } & Left radial artery & $35(13.3)$ & $10(28.6)$ & $25(71.4)$ & & \\
\hline & Right dorsalis pedis artery & $3(1.1)$ & 0 & $3(100)$ & & \\
\hline \multirow{2}{*}{ History of smoking } & Yes & $192(72.7)$ & 42 (21.9) & $150(76.4)$ & 0.9111 & 0.763 \\
\hline & No & $72(27.3)$ & $17(23.6)$ & $55(76.4)$ & & \\
\hline \multirow{2}{*}{ History of diabetes } & Yes & $72(27.3)$ & $11(15.3)$ & $61(84.7)$ & 2.8521 & 0.091 \\
\hline & No & $192(72.7)$ & $48(25.0)$ & $144(75.0)$ & & \\
\hline \multirow{2}{*}{ History of drinking } & Yes & $191(72.3)$ & $45(23.6)$ & $146(76.4)$ & 0.5841 & 0.445 \\
\hline & No & $73(27.7)$ & $14(19.2)$ & $59(80.8)$ & & \\
\hline \multirow[t]{2}{*}{ History of coronary heart disease } & Yes & $154(58.3)$ & $32(20.8)$ & $122(79.2)$ & 0.5241 & 0.469 \\
\hline & No & $110(41.7)$ & $27(24.5)$ & $83(75.5)$ & & \\
\hline \multirow{2}{*}{ History of hypertension } & Yes & $137(51.9)$ & $28(20.4)$ & $109(79.6)$ & 0.5991 & 0.439 \\
\hline & No & $127(48.1)$ & $31(24.4)$ & $96(75.6)$ & & \\
\hline \multirow{2}{*}{ Use of analgesics } & Yes & $182(68.9)$ & $16(8.8))$ & $166(91.2)$ & 62.061 & $<0.001^{*}$ \\
\hline & No & $82(31.1)$ & $43(22.3)$ & $39(47.6)$ & & \\
\hline \multirow{2}{*}{ Use of sedatives } & Yes & $59(22.3)$ & $21(24, .1)$ & $38(21.5)$ & 0.2391 & 0.625 \\
\hline & No & $205(77.7)$ & $66(32.2)$ & $139(67.8)$ & & \\
\hline \multirow{2}{*}{ Use of protective catheter sleeves } & Yes & $168(63.6)$ & $31(18.5)$ & $137(81.5)$ & 4.0411 & $0.044 *$ \\
\hline & No & $96(72.4)$ & $28(29.2)$ & $68(70.8)$ & & \\
\hline \multirow{2}{*}{ Use of restraints } & Yes & $181(68.6)$ & $31(17.1)$ & $150(73.2)$ & 9.0441 & $0.003 *$ \\
\hline & No & $83(31.4)$ & $28(47.5)$ & $55(26.8)$ & & \\
\hline
\end{tabular}

Note: $\left.{ }^{*} \mathrm{P}<0.05 ; 1\right)$ is the $\chi^{2}$ value; 2 ) is the t value 


\section{Results}

\subsection{The Status of Catheter Failure}

In the present study group, 59 (22.34\%) out of 264 patients had catheter failure, of which 47 patients $(17.8 \%)$ had edema around the puncture site, 12 patients $(4.5 \%)$ had spasm in the affected limb, 10 patients $(3.8 \%)$ had skin bruising around the puncture site, 29 patients $(10.8 \%)$ had bleeding around the puncture site, 29 patients $(8.5 \%)$ had catheter oozing, 6 patients $(2.3 \%)$ had catheter buckling, and 18 patients $(2.8 \%)$ had catheter blockage.

\subsection{Single Factor Analysis of the Catheter Failure}

Table 1 shows the single factor analysis of catheter failure in patients. In a total of 264 patients single factor analysis showed that the use of analgesics, protective catheter sleeves, and affected limb restraints were significantly associated with the catheter failure $(\mathrm{P}<0.05)$.

\subsection{Multivariate Analysis of the Catheter Failure}

The variables with statistical significance in the single factor analysis: the use of analgesics, the use of protective catheter sleeves, and the use of affected limb restraints $(\mathrm{P}<0.05)$ were included in the multivariate logistic regression model. Table 2 shows the variables of the multivariate logistic regression. The forward Wald procedure was used and the result showed that the use of affected limb restraints, and the use of analgesics were the protective factors in preventing the catheter failure $(\mathrm{P}<0.05)$. Table 3 shows multivariate regression of factors involved in catheter failure.

Table 2. Values of independent variables.

\begin{tabular}{ll}
\hline Independent variables & Values \\
\hline Use of sedatives & $1=$ Yes, $2=$ No \\
Use of analgesics & $1=$ Yes, $2=\mathrm{No}$ \\
Use of protective catheter sleeves & $1=$ Yes, $2=\mathrm{No}$ \\
Use of affected limb restraints & $1=$ Yes, $2=\mathrm{No}$ \\
\hline
\end{tabular}

Table 3. Multivariate logistic regression of the catheter failure.

\begin{tabular}{llllll}
\hline Variables & $\boldsymbol{\beta}$ value & SE & Wald $\chi^{2}$ & value & P value \\
\hline Constants & 4.364 & 0.863 & 23.547 & $<0.001$ & OR value (95\%CI) \\
Use of restraints & -0.770 & 0.357 & 4.650 & 0.031 & $0.463(0.230-0.932)$ \\
Use of analgesics & -3.133 & 0.473 & 43.904 & $<0.001$ & $0.044(0.017-0.110)$ \\
\hline
\end{tabular}

\section{Discussion}

\subsection{Analysis of the Causes of the Failure of the Invasive Ultrasound-Guided Arterial Catheterization}

Ultrasound-guided invasive arterial catheterization avoids pain, and vascular damage caused by repeated puncture and related other complications, however many other factors can lead to catheter failure during the procedure. The results of this study showed that the catheter failure occurred in 59 cases, and the failure rate is $22.34 \%$, which is slightly lower than that is reported by Wang Xiaorong et al. [9] The incidence of affected limb edema is $17.8 \%$, and the incidence of puncture site bleeding was $10.8 \%$, which were significantly lower than that is reported by Wang Juan et al. [10] To avoidthe possible diversity due todifferent methods and techniques, all 264 patients in this group were subjected to catheterization by the same doctor, with a strict catheterization training. The Supertach5 the latest professional vascular access catheter was used as the puncture needle. The Supertach5 puncture needle is a safe indwelling needle with double protection against reflux, which can prevent blood reflux and effectively avoid catheter-related infections. Its puncture needle tip can be developed under color ultrasound, which makes it easier for the operator to determine the position of the needle tip and control the needle direction to avoid blood vessel perforation. The needle cannula is soft, has little irritation to the blood vessel after indwelling, and is convenient to be fixed after the catheter is placed, which increases the patient's comfort.
Therefore, it is recommended that medical staff who use invasive arterial catheters should receive strict training, try to choose safe arterial puncture needles and standardize the catheterization process and catheter fixation methods, which can improve the success rate of invasive catheter placement and reduce the incidence of catheter failure.

In this study, the incidences of oozing and catheter blockage were $2.8 \%$ and $8.5 \%$, respectively, which may be related to the formation of a certain pressure on the catheter due to the subcutaneous leakage caused by the patient's hypoproteinemia. Most patients in the present study were middle-aged and elderly patients, who were in critical condition. The disease itself is complicated, and at the same time, it is often combined with various factors such as associated other diseases, multiple organ failure, internal environment disorders, which may result in hemodynamics. Together with hyperviscosity, it is easier to block the catheter after catheterization. Patients with coagulopathy are more prone to have oozing and bleeding around the puncture site. Elderly patients have less subcutaneous fat, increased blood vessel fragility, and poor skin elasticity, which may cause catheter failure, and it is basically consistent with the results of Nie Liang et al. [11-13]

\subsection{Effective Limb Restraint Is a Protective Factor Against the Catheter Failure}

The restraint belt protects the patient, prevents the patient from hurting himself or others, and protects the patient's safety and treatment effect by restricting the patient's physical activity. Protective restraint is one of the important 
nursing measures in clinical nursing, and it has a significant advantage in reducing the occurrence of unplanned extubation and other adverse events caused by agitation [14, 15]. ICU patients are in a critical condition, and their consciousness is affected to a certain extent. If they are not given protective restraints before a full recovery, the probability of unplanned extubation and catheter prolapse is greatly increased, which will affect the treatment effect [16-19]. Effective limb restraint plays an important role in preventing unplanned extubation, but it can also cause some adverse reactions to patients [20-22]. The results of this study showed that the incidence of affected limb edema was $17.8 \%$, and edema may involve a variety of pathophysiological mechanisms and is related to a variety of risk factors, such as reduced muscle activity, slow blood flow, poor blood reflux, and internal environment disorders, etc., which were caused by the reduced limb activity after applying the limb restraints, and this is consistent with the results of Wang Caihong et al. [23] Therefore, if the condition of critically ill-patients is relatively safe, ICU physicians, neurologists, rehabilitation therapists, who participate in the management and intervention of critical patients, should find a relative balance between the physical restraints, and the patients' activities, and conduct appropriate physical rehabilitation training to prevent the occurrence of complications [22].

\subsection{Using Protective Catheter Sleeves Can Reduce Catheter Failure Rate}

Improper supervision and poor fixation after catheter placement will increase catheter failure [24-27]. Self-designed protective catheter sleeves were used for some patients in this study. The results showed that under the synergistic effect of using analgesics, and effective limb restraints, the catheter failure rate of patients using the protective catheter sleeves was significantly lower than that of patients who did not use the protective sleeves. The protective sleeve was designed by our department. The forearms, and palms of the protective sleeve is used to prevent excessive activity of the wrist joint and gliding and buckling of the catheter. The protective sleeve is provided with a puncture port skin observation window to observe the skin condition of the puncture port after the catheter is placed and observe the catheter failure in time. The protective sleeve is easy to put on and take off, will not cause discomfort to the arm or skin crush, and can be repeatedly used after sterilization. As an enhanced protective device for invasive catheters, this sleeve can prevent the arterial catheter slippage safely and effectively.

\subsection{Using Analgesics Can Reduce Catheter Failure Rate}

ICU patients generally suffer from pain, which has become the fifth vital sign after temperature, pulse, breathing, and blood pressure [28]. The results of this study showed that the rate of invasive catheter failure in patients using analgesics was significantly lower than that in patients not using effective analgesics, which is consistent with the study reported by Zhang Haiyan et al. [29] The possible reason is the postoperative and post-traumatic acute pain causes stress in the body, increases secretion of catecholamines and other substances in the blood vessels, subsequently results in internal environment disorders, reduced overall body comfort, decreased immunity, and infection that reduce patient' comfort, and cause restlessness, which can easily lead to catheter failure. Therefore, pain control plays an important role in ICU patients.

\section{Conclusion}

Invasive arterial catheterization is of great significance for the dynamic observation of critically ill-patients. The effective maintenance of the catheter and preventing its failure is the focus of nursing. The results of this study indicate that puncture limb restraining, the use of protective catheter sleeves, and the use of appropriate analgesics can effectively reduce the failure rate of invasive arterial catheters. Preoperative patient education, standardized operational norms, selection of safe and appropriate puncture needles, postoperative effective restriction of the affected limbs, use of protective catheter sleeves, use of analgesics and sedatives, and psychological nursing are very important for the prevention of invasive arterial catheter failure.

\section{References}

[1] Chen Yi, Wu Lei. Effect evaluation of ultrasound-guided puncture in establishing invasive blood pressure pathway in ICU. Contemporary Medical Symposium. 2020; 18 (1): 267-268.

[2] Peng Yan, Wang Junying, Peng Xuegang, Wen Gang, Qiao Ming, Liu Jie. Study on the invasive arterial pressure monitoring in acute and critical patients. Chinese Community Doctors. 2015; 31 (33): 110-112.

[3] LaRue GD. Efficacy of ultrasonography in peripheral venous cannulation. J Intraven Nurs. 2000; 23 (1): 29-34.

[4] Ye Hongbo, Qian Qindan, Zhang Xiaochun, Zhang Shaoli, Shi Xiuxia. Application of ultrasound-guided radial artery puncture and catheterization in patients with hemorrhagic shock. Chinese Journal of Rural Medicine and Pharmacy. 2015; 22 (17): 22-23.

[5] Huang Limin. Causes and nursing strategies of complications in invasive arterial pressure monitoring. Journal of Nursing and Rehabilitation. 2008; 7 (2): 110-111.

[6] Wu Jiamin, Jiang Zhiping, Jiang Yuqin. Observation on the application of ultrasound-guided invasive arterial puncture and catheterization in traumatic shock patients. Journal of Nursing and Rehabilitation. 2018; 7 (2): 66-67.

[7] Nie Liang, Liu Xiaofeng, Xie Hongying, et al. Application of B-ultrasound guided arterial catheterization in ICU shock patients. Contemporary Medicine. 2017; 23 (17): 5-7.

[8] Liu Wencai, Wang Fengyun, Zhou Ailing, Li Mingzhen. Factors affecting the accuracy of invasive arterial blood pressure and nursing strategies. Chinese Journal of Practical Nursing. 2006; 22 (5): 48. 
[9] Wang Xiaorong, Liang Rong, Yuqin, Zhao Defeng, Xu Zhi. Application of quality control ring to reduce the failure rate of peripheral invasive arterial catheters. Journal of Clinic Nursing's Practicality. 2018; 3 (49): 100-103.

[10] Wang Juan. Application of invasive blood pressure monitoring by catheterization of radial artery and posterior tibial artery in critically ill neonates. Infection International (Electronic Version). 2020; 9 (1): 105.

[11] Powell JT, Mink JT, Nomura JT, et al. Ultrasound-guidance can reduce adverse events during femoral central venous cannulation. J Emerg Med. 2014; 46 (4): 519-24.

[12] Troianos CA, Hartman GS, Glas KE, et al. Guidelines for performing ultrasound guided vascular cannulation: recommendations of the American Society of Echocardiography and the Society of Cardiovascular Anesthesiologists. J Am Soc Echocardiogr. 2011; 24 (12): 1291-318.

[13] Wang Xiaorong, Liang Rong, Yu Qin, Zhao Defeng, Xu Zhi. Analysis and strategies of bleeding-related factors in puncture site after transradial PCI. Journal of Clinic Nursing's Practicality. 2017; 2 (29): 174-176.

[14] Zhu Chunmin. Application of humanistic nursing model in psychiatric nursing. Journal of Clinical Psychosomatic Diseases. 2016; 22 (z1): 234-235.

[15] Zhu Shengchun, Jin Yumei, Xu Zhihong, Xia Fengying, Luo $\mathrm{Na}$. Analysis of the characteristics of physical restraint usage and nursing status of ICU patients. Chinese Journal of Nursing. 2009; 44 (12): 1116-1118.

[16] Xie Chen. Application value of humanized protective restraints in ICU nursing management. Journal of clinical medical literature. 2020; 7 (30): 108.

[17] Li Na, Xing Yan, Zhang Shanshan, Wang Shulian. Application value of humanized protective restraints in ICU nursing management. Journal of clinical medical literature. World Latest Medicine Information (Continuous electronic journal). 2018; 18 (5): 250.

[18] Wang Yingying, Song Yan. Observation on the application effect of humanized protective restraints in ICU nursing. Journal of clinical medical literature. 2019; 6 (1): 125-126.

[19] Li Jiaxing, Ren Zhijun, Zhang Zijun, Cheng Anqi, Zhang
Tingting, Xia Xinhua. Effects of comprehensive restraint reducing program on unplaned extubation among ICU patients with tracheal intubation. Chinese Journal of Nursing. 2017; 52 (5): 549-553.

[20] Wang Guoying, He Xiaoci, Gao Mengying, Ma Yanxin, Tian Jiao, Tian Suzhai. Application of continuous quality improvement in physical restraint management. Chinese Journal of Practical Nursing. 2016; 32 (11): 840-843.

[21] Qu Ruyi, Fu Xiaoyan. The effect of nursing interventions combined with programmed sedation and analgesia on ICU patients. Electronic Journal of Practical Clinical Nursing Science 2019; 4 (26): 21.

[22] Chen Zhilin, Chen Xi, Zheng Panpan, Chen Peiying, Meng Liping. The effect of early progressive rehabilitation training on the recovery of neuromuscular function in critically ill patients with mechanical ventilation. Medical Diet and Health. 2020; 18 (9): 11-13.

[23] Wang Caihong, Qin Junmei, Ben Yanli. Early rehabilitation training improves ICU acquired muscle weakness in patients with mechanical ventilation. Chinese Nursing Management. 2019; 19 (3): 457-461.

[24] Zhang Jing. Analysis of the causes of accidental extubation of invasive arterial blood pressure monitoring and nursing strategies. Chinese General Practice Nursing. 2015; (25): 2493-2495.

[25] Liang Qing, Yang Ning. Discussion on quality control circle activity in reducing accidental extubation of central venous catheterization. Chinese Medical Innovations. 2014; (13): 95-97.

[26] Wu Xiaolian. Analysis of the causes of accidental extubation of deep vein catheterization and nursing strategies. Journal of Nursing and Rehabilitation. 2009; 8 (10): 859-860.

[27] Zhou Lijin. Analysis of the causes of unplanned extubation of ICU patients with tracheal intubation and nursing strategies. Medical Information (Early Issue). 2010; 23 (9): 3170-3171.

[28] Chen Jie, Zhang Haiyan, Wu Xiaoying, Lu Qian. Research progress of objective pain assessment in adult critically ill patients. Chinese Journal of Nursing. 2014; 49 (3): 355-359.

[29] Zhang Haiyan, Chen Jie, Wu Xiaoying, et al. Survey of pain nursing management status in 40 hospitals of China. Chinese Nursing Management. 2014; (11): 1121-1124. 